\title{
Anemia in the ICU: are your patients needin' erythropoetin?
}

\author{
David Hecht and Arthur Boujoukos* \\ University of Pittsburgh Department of Critical Care Medicine: Evidence-Based Medicine Journal Club, edited by Sachin Yende
}

\author{
Extended abstract \\ Citation \\ Corwin HL, Gettinger A, Fabian TC, May A, Pearl RG, \\ Heard S, An R, Bowers PJ, Burton P, Klausner MA, and \\ Corwin MJ, for the EPO Critical Care Trials Group: \\ Efficacy and Safety of Epoetin Alfa in Critically Ill \\ Patients N Engl J Med 2007;357:965-76
}

\section{Background}

Anemia, which is common in the critically ill, is often treated with red-cell transfusions which are associated with poor clinical outcomes. We hypothesized that therapy with recombinant human erythropoietin (epoetin alfa) might reduce the need for red-cell transfusions.

\section{Methods \\ Objective: Evaluate the efficacy of epoetin alfa in reducing the packed red blood cell transfusion requirement in critically ill patients. \\ Design: Prospective, randomized, double blind, placebo controlled, multi-center trial \\ Setting: 115 medical centers throughout the United States. Subjects: 1460 patients that had been admitted to medical, surgical, or trauma intensive care units. Inclu- sion criteria included age 18 years or greater, patients remained in the ICU for two days, and hemoglobin level less than $12 \mathrm{gm} / \mathrm{dl}$. Statistical analysis was performed for the entire group, as well as subgroup analysis according to the type of ICU to which the patient was admitted (the subgroup analysis was included in the initial study design). \\ Intervention: After enrollment, patients randomized to the study group received epoetin alfa 40,000 units SQ on study day 1,8 , and 15 . The drug was withheld if the hemoglobin level was greater than $12 \mathrm{gm} / \mathrm{dl}$ at the time of scheduled administration. Patients randomized to the}

*Correspondence: boujoukosaj@upmc.edu

Department of Critical Care Medicine, 612A Scaife Hall, 3550 Terrace Street, University of Pittsburgh, Pittsburgh, PA 15261, USA placebo group received a placebo injection according to the same schedule. All patients enrolled received supplemental iron. Patients were given packed red blood cell transfusions at the discretion of the treating physician. The investigators recommended goal hemoglobin concentration of 7 to $9 \mathrm{gm} / \mathrm{dl}$, and not to transfuse for a hemoglobin concentration of greater than $9 \mathrm{gm} / \mathrm{dl}$ unless a specific indication was present.

Outcomes: The primary endpoint was percentage of patients requiring blood transfusion. Secondary endpoints included number of units transfused, mortality, and the change in hemoglobin concentration from baseline.

\section{Results}

As compared with the use of placebo, epoetin alfa therapy did not result in a decrease in either the number of patients who received a red-cell transfusion (relative risk for the epoetin alfa group vs. the placebo group, 0.95; $95 \%$ confidence interval [CI], 0.85 to 1.06 ) or the mean $( \pm$ SD) number of red-cell units transfused $(4.5 \pm 4.6$ units in the epoetin alfa group and $4.3 \pm 4.8$ units in the placebo group, $\mathrm{P}=0.42$ ). However, the hemoglobin concentration at day 29 increased more in the epoetin alfa group than in the placebo group $(1.6 \pm 2.0 \mathrm{~g}$ per deciliter vs. $1.2 \pm 1.8 \mathrm{~g}$ per deciliter, $\mathrm{P}<0.001)$. Mortality tended to be lower at day 29 among patients receiving epoetin alfa (adjusted hazard ratio, $0.79 ; 95 \% \mathrm{CI}, 0.56$ to 1.10 ); this effect was also seen in pre-specified analyses in those with a diagnosis of trauma (adjusted hazard ratio, 0.37; 95\% CI, 0.19 to 0.72 ). A similar pattern was seen at day 140 (adjusted hazard ratio, 0.86; 95\% CI, 0.65 to 1.13), particularly in those with trauma (adjusted hazard ratio, $0.40 ; 95 \% \mathrm{CI}, 0.23$ to 0.69 ). As compared with placebo, epoetin alfa was associated with a significant increase in the incidence of thrombotic events (hazard ratio, 1.41; $95 \%$ CI, 1.06 to 1.86 ).

\section{Conclusions}

The use of epoetin alfa does not reduce the incidence of red-cell transfusion among critically ill patients, but it may reduce mortality in patients with trauma. Treatment with epoetin alfa is associated with an increase in the 
incidence of thrombotic events. (ClinicalTrials.gov number, NCT00091910.)

\section{Commentary}

Anemia is a common problem in critically ill patients, frequently requiring packed red blood cell transfusion. Transfusions are associated with potential complications (hemolytic transfusion reactions, non-hemolytic transfusion reactions, infectious disease transmissions). Previous studies investigated the administration of human recombinant erythropoietin to critically ill patients to reduce transfusion requirements. The first of these studies demonstrated fewer transfusions with the administration of erythropoietin, though there was no difference in mortality. ${ }^{1}$ A similar, larger study was performed by many of the same researchers finding a similar decrease in transfusion requirements in the treatment group and no overall mortality difference. ${ }^{2}$ Additionally, in a post hoc subgroup analysis, they identified a mortality benefit in trauma patients.

The current article was performed in order to investigate additional outcomes, primarily the safety of epoetin alfa for this indication. The study design was virtually the same as the 2002 study, although the current study evaluates mortality benefit at 140 days (an extended time period from conventional 28-day mortality), and predetermined subgroup analysis of medical, surgical, and trauma ICU patients. It is interesting that the findings of this study do not reflect the transfusion benefit previously demonstrated.

The current trial shows no difference in the PRBC transfusion requirements, no clinically significant difference in hemoglobin concentration (though there is a statistical difference), and no mortality benefit. So, what is the significance of this study? The importance of this study lies within the predetermined subgroup analysis. In these analyses, mortality benefit was observed in the trauma patients, both at 29 days (hazard ratio $[\mathrm{HR}]=0.37$, CI:0.19 - 0.72) and at 140 days (HR=0.40, CI: $0.23-$ 0.69 ). The mortality benefit found in this data is not new. Similar findings were shown in the previous trial, where a post hoc subgroup analysis was performed in trauma patients and mortality benefit was observed at 29 days $(\mathrm{HR}=0.43$, CI: $0.23-0.81)$. Thus, two large studies have now shown a mortality benefit. However, the causal mechanisms are unclear. The benefit does not correspond to improvement in hemoglobin concentration or reduction in transfusion requirement. The authors speculate that it may be related to non-erythrogenic properties of epoetin alfa, such as anti-apoptotic characteristics or protection against hypoxemia.

The safety evaluation in this trial showed that epoetin alfa increases the incidence of clinically relevant thrombotic events ( 16.5 vs. $11.5, \mathrm{p}=0.008)$. These findings, however, are not entirely conclusive. With further analysis, the authors showed that the increase in thrombotic events only affected patients not receiving heparin prophylaxis. The incidence of thrombotic events was 20.3 vs. 12.8 $(\mathrm{p}=0.008)$ in patients not receiving prophylaxis, and 12.3 vs. $10.2(\mathrm{p}=0.41)$ in patients receiving prophylaxis.

As with previous trials, this is a large, multi-center, prospective, blinded, placebo-controlled trial. Why then is the transfusion requirement benefit not reproduced in this study? This difference is likely related to a change in transfusion culture. In 1999, the TRICC trial was published, and this study showed that a restrictive transfusion approach had similar outcomes compared to liberal transfusion strategy. ${ }^{3}$ Previous trials recruited patients prior to these findings, whereas the current study recruited patients after dissemination of findings of the TRICC study. The lack of differences in transfusion requirement in this study may have occurred due to less aggressive transfusion practices.

With the current findings, it is evident that epoetin alfa administered to critically ill patients does not improve transfusion requirements as previously demonstrated. The mortality benefit shown in trauma patients should be considered, as this benefit has been found independent of transfusion requirement and hemoglobin concentrations in two well designed trials. Mechanisms underlying this beneficial effect should be examined in future studies. It is likely that epoetin alfa can be administered safely to critically ill patients that are also receiving heparin in prophylactic doses.

\section{Recommendations}

Epoetin alfa should not be used in critically ill patients to decrease PRBC requirements. Epoetin alfa can be considered in critically ill trauma patients as there is a demonstrated mortality benefit, but these patients should be able to safely receive prophylactic heparin.

Competing interests

The authors declare that they have no competing interests.

Published: 17 December 2010

References

1. Corwin HL, Gettinger A, Rodriguez RM, Pearl GR, Guble KD, Enny C, Colton T, Corwin MJ: Efficacy of recombinant human erythropoietin in the critically ill patient: A randomized, double-blind, placebo-controlled trial. Critical Care Medicine 1999, 27: 2346-2350

2. Corwin HL, Gettinger A, Pearl GR, Fink MP, Levy MM, Shapiro MJ, Corwin MJ, Colton TC: Efficacy of Recombinant Human Erythropoietin in Critically III Patients: A Randomized Controlled Trial. JAMA 2002, 288: 2827-2835

3. Herbert PC, Wells G, Blajchman MA, Marshall J, Martin C, Pagliarello G, Tweeddale M, Schweitzer I, Yetiser E: A Multicenter, Randomized, Controlled Clinical Trail of Transfusion Reuirements in the Critically III. NEJM 1999, 340: 409-417

doi:10.1186/cc9368

Cite this article as: Hecht D, Boujoukos A: Anemia in the ICU: are your patients needin' erythropoetin? Critical Care 2010, 14:332. 